\title{
Original
}

CODEN-JHTBFF, ISSN 1341-7649

\section{Fluoride Supplement Affects Bone Mineralization in Young Rats}

\author{
Masahisa Inoue ${ }^{1,2)}$, Racquel Z. LeGeros' ${ }^{2}$, Miho Inoue ${ }^{2)}$, Rosario Santos Rivera' ${ }^{1)}$, Gulsan Ara Sathi ${ }^{1)}$, \\ Hidetsugu Tsujigiwa, Hitoshi Nagatsuka ${ }^{3)}$, Masahiko Akita ${ }^{1)}$ and Kojun Setsu ${ }^{1)}$
}

\author{
${ }^{1}$ Laboratories for Structure and Function Research, Faculty of Pharmaceutical Sciences, Tokushima Bunri University, Tokushima \\ 770-8514 Japan \\ ${ }^{2}$ Department of Biomaterials and Biomimetics, College of Dentistry, New York University, New York 10012 USA \\ ${ }^{3}$ Department of Oral Pathology and Medicine, Graduate School of Medicine, Dentistry and Pharmaceutical Sciences, Okayama \\ University, 700-8525, Japan \\ (Accepted for publication, March 25, 2006 )
}

\begin{abstract}
Fluoride as a supplement can affect the structural integrity of bone. Fluoride that is incorporated in the mineral, substitutes for the hydroxyl group producing hydroxyfluorapatite crystals and presumed to increase bone strength by preventing resorption. Because of this, fluoride therapy has been carried out in clinical trials for the treatment of osteoporosis. Although fluoride renders the mineralized tissues resistant to resorption, the effects of fluoride on bone strength and cortical bone mass specially that of the developing bone has not yet been characterized. The purpose of the study was to analyze the effect of fluoride on developing teeth and bone in young rats through histomorphometry. Sixteen wistar rats were used and divided into two groups; nonfluoride supplemented (control) and fluoride-supplemented groups. Four weeks after, the rats were sacrificed and the mandible and femur were subjected to histological and histomorphometric analyses. No clinical difference was observed between the two groups. The amount of mineralized cartilage was higher in fluoride-supplemented group compared to the control group. However, the amount of new bone was lesser in fluoride-supplemented group compared to the control group. This histomorphometric analysis showed that during endochondral ossification, 10 ppm fluoride administration caused some changes in calcified cartilage and bone mineralization in young rats.
\end{abstract}

Keywords: Fluoride, Mineralization, Histomorphometry

\section{Introduction}

It has been recognized that fluoride affects the mineralization of bone and teeth. Fluoride is usually incorporated in the mineral, and increases the resistance of bone to acid dissolution thereby reducing bone resorption. However, the advantage of fluoride greatly depends on the amount administered ${ }^{1)}$. Fluoride supplement has been reported to affect bone mineralization in various ways. At all doses, fluoride has physicochemical effect on bone. As it is incorporated in the mineral, it substitutes for the hydroxyl groups in the carbonate-apatite structure to produce hydroxyfluorapatite.

Experiments on fluoride therapy for the treatment of osteoporosis started during the 1960's ${ }^{2}$. Although fluoride supplement has been regarded as advantageous to the mineralization of hard tissues, high dose administration has detrimental effects. The potency of fluoride therapy is perplexed by the bi-phasic dose relationship as well as the difficulty in determining the appropriate dosage ${ }^{3)}$. Some studies showed that

Corresponding author:Inoue Masahisa.Laboratories for Structure and Function Research, Faculty of Pharmaceutical Sciences, Tokushima Bunri University, Tokushima 770-8514 Japan, low doses of fluoride stimulate bone formation without altering bone formation ${ }^{4,5)}$. On the other hand, high dosage of fluoride did not decrease the spinal fracture rate and it increased the rate of microfractures ${ }^{6}$. At higher doses, fluoride is thought to have an effect on bone cells: mitogenic to osteoblasts and inhibitory to osteoclasts ${ }^{7}$. Others reported that fluoride intake at high levels had no negative effect on bone mineralization. Fluoride intake was associated with slight increases in trabecular bone volume and trabecular thickness but these effects could not be demonstrated consistently ${ }^{8)}$. Furthermore, fluoride directly affects cell proliferation and alkaline phosphatase activity in culture study ${ }^{9}$. Nevertheless, it is not known whether fluoride has an effect on the recruitment and activation of osteoblasts in vivo. Few studies have been conducted regarding the histomorphometric analysis of fluoride-treated bone and cell ${ }^{10,11}$. These studies were concerned with osteoporosis and used adult or old animals.

In line with this, we analyzed the effect of fluoride on developing teeth and bone in young rats through histological and histomorphometric analyses.

Materials and methods 


\section{Animals}

Sixteen four week-old, male wistar rats were randomly divided into 2 groups. All animals were housed in plastic cages, maintained in an air-conditioned laboratory having a 12-hour automatic lighting and fed regularly with rodent diet containing $0.74 \%$ calcium, $0.15 \%$ magnesium and $0.74 \%$ phosphorus. The nonfluoride supplemented group (control) received distilled water and the fluoride-supplemented group received $10 \mathrm{ppm}$ sodium fluoride water solution $(0.52 \mathrm{mM} / 1 \mathrm{NaF})$. The body weight, feed and fluid consumption were monitored everyday for 4 weeks. On day 28 following an overnight fast, the rats were euthanized with $\mathrm{CO}_{2}$ anesthesia. After confirmation of euthanasia by eye color and lack of respiration, the mandibular bone and femur were dissected. The samples were immersed in 4\% paraformaldehyde solution in preparation for histological analysis.

\section{Histomorphometry}

The fixed mandibles and femur bones were decalcified with 10\% EDTA solution for 3 weeks. The specimens were embedded in paraffin blocks in a routine manner, sectioned longitudinally into $4 \mathrm{~m}$ thickness and stained with hematoxylin and eosin. The stained sections were observed under light microscopy and analyzed by using the Bioquant Image Analyzer ( $R$ and $M$ Biometrics, Nashville, TN, USA). The amount of new bone, mineralized cartilage and the cartilage/bone ratio were measured at the metaphysis beside chondro-osseous junction of femur and expressed in percentages.

\section{Results}

\section{Clinical growth and mineral consumption}

The animals were monitored daily during the entire 4-week experimental period. None of the experimental animal died. No clinical difference was observed in both groups. The mean initial weight, weight gained, feed and fluid consumptions were determined for both groups and statistically analyzed. There was no significant effect of fluoride observed clinically. All data were represented as \pm S.E.M. (Table 1 ).

\section{Histology and histomorphometry}

The trabeculae showing mineralized cartilage in fluoride-

Table 1. Comparison between the control and fluoride-supplemented group

\begin{tabular}{lcc}
\hline & Control & Fluoride supplement \\
\hline Initial weight $(\mathrm{g})$ & $121.6 \pm 2.7$ & $124.2 \pm 4.9$ \\
Weight gained $(\mathrm{g})$ & $210.2 \pm 19.4$ & $220.8 \pm 19.9$ \\
Feed consumption (g) & $1162.2 \pm 65.8$ & $1256.0 \pm 70.1$ \\
Fluid consumption (ml) & $665.1 \pm 56.6$ & $683.7 \pm 33.7$ \\
Fluoride consumption & $0.0 \pm 0.0$ & $2.09 \pm 0.03$ \\
(mg/kg bw/day) & & \\
\hline
\end{tabular}

Data were obtained from the means of \pm S.E.M of eight rats per group.
Table 2. Histomorphometrical analysis

\begin{tabular}{lcc}
\hline & Control & Fluoride supplement \\
\hline New bone/ total area & $56.2 \pm 3.5$ & $46.0 \pm 4.6^{*}$ \\
Mineralized cartilage/ total area & $18.0 \pm 1.7$ & $24.9 \pm 5.0^{*}$ \\
Cartilage/ bone ratio & $32.6 \pm 3.1$ & $52.6 \pm 5.8^{* *}$ \\
\hline
\end{tabular}

Data were obtained from the means of \pm S.E.M of eight rats per group. $* \mathrm{P}<0.05$ vs. control $* * \mathrm{P}<0.01$ vs. control

supplemented group were wider and thicker compared to the control group (Fig. 1). Few mineralized cartilage within the cortical bone was also observed (Fig. 2). Histomorphometric analysis of the endochondral ossification of the femur showed that the amount of new bone was higher in the control group compared to the fluoride- supplemented group. On the other hand, the amount of mineralized cartilage was higher in fluoride-supplemented group than in the control group. The cartilage/bone ratio was higher in

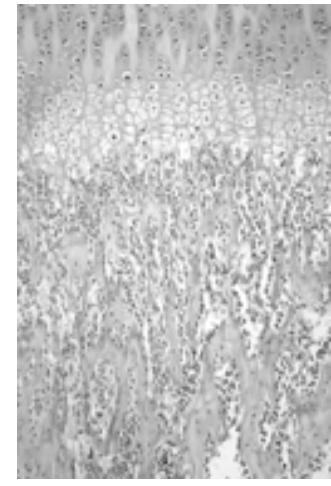

Fig. 1a

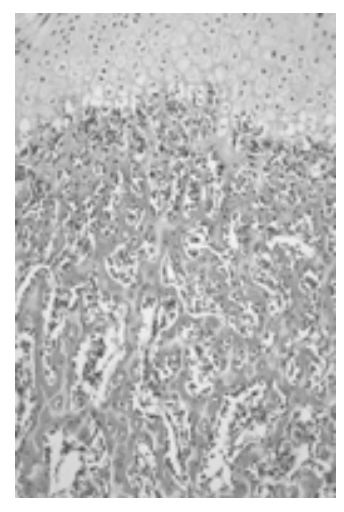

Fig. 1b
Fig. 1. Light micrographs of femur showing endochondral ossification in control group (a) and fluoride-supplemented group (b). Mineralized cartilage trabeculae in fluoride-supplemented group are wider than the control group ( $\mathrm{H}$ and $\mathrm{E}$ ).

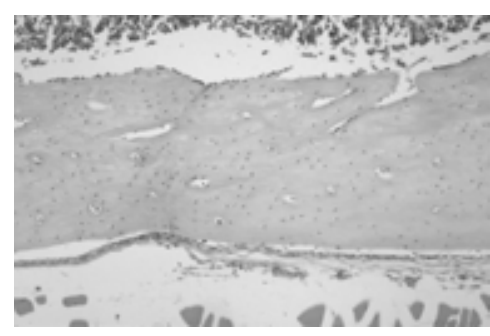

Fig. 2a

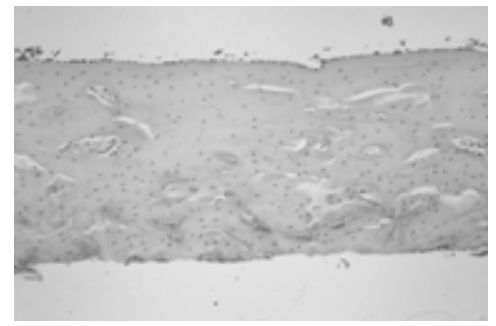

Fig. 2b

Fig. 2. Light micrographs of the cortical bone of control (a) and fluoridesupplemented group (b). Few mineralized cartilages were observed within the cortical bone in fluoride-supplemented group ( $\mathrm{H}$ and $\mathrm{E}$ ). 
Masahisa Inoue et al.: Fluoride Supplement Affects Bone Mineralization

fluoride-supplemented group compared to the control group. Table 2 shows the results of the histomorphometric analysis of both groups. No significant change on tooth structures was seen in fluoride-supplemented group (data not shown).

\section{Discussion}

The effect of fluoride on bone and tooth mineralization has been well recognized. Fluoride is only one of the few known agents that can stimulate osteoblast proliferation and increase new mineral deposition in cancellous bone. Fluoride incorporation into the bone increases the size and thus decreases the solubility of the bone apatite crystals ${ }^{1)}$. It has been used clinically for the prevention and treatment of osteoporosis although controversies still exist. Therapeutic future for sodium fluoride in osteoporosis lies with early administration and low-dose regimens in which toxic levels are avoided and mineralization is not impaired ${ }^{12)}$.

In this study, histomorphometric analysis of endochondral ossification showed that fluoride supplement increased the amount of mineralized cartilage. During endochondral ossification, hypertrophic chondrocytes alter the matrix they produce by adding type $\mathrm{X}$ collagen and fibronectin to enable it to become mineralized by calcium carbonate. It has also been recognized that resorption of hard tissue is mandatory for bone growth and remodeling ${ }^{13)}$. The fluoride was incorporated in the early stage of bone development and that was during endochondral ossification. The incorporation of fluoride affected the mineralization of cartilage by increasing the cartilage mass.

Although there was an increased in the amount of mineralized cartilage, the amount of new bone was reduced histologically and histomorphometrically. The reduction in the amount of new bone may be due to the delay in the deposition of mineralized bone matrix following the death of the chondrocytes. This result coincided with previous reports that bone mineralization is delayed by fluoride ${ }^{14}$. Fluoride may stimulate bone formation but it also prolongs the time period of mineralization ${ }^{15)}$. This was also reported in one study where in high fluoride intake caused osteomalacia and diminished bone strength in rats ${ }^{16)}$. The evidence also indicates that fluoride produces bone mineral with intrinsically weaker material properties than normal mineral. This could be related to one study where in fluoride may have some effects on the organic matrix like in the post-translational assembly of the glycosaminoglycan chains which may be an influential factor in the mineralization process ${ }^{17}$. Fluoride can augment the rate of mineral deposition by reducing the solubility of precipitating fluoridated apatites ${ }^{18)}$.

It has been reported that treating both rats and rabbits with fluoride was found to decrease bone strength despite increases in bone mass and bone volume fraction ${ }^{19)}$. Also, high fluoride intake had no effect on trabecular bone volume but instead increased the amount of unmineralized osteoid particularly in older rats ${ }^{20)}$. Although lesser amount of new bone mass in fluoride- supplemented group was obtained in this study, clinically, there was no difference between the two groups. Unfortunately bone quality assessment was not done.

Furthermore, some mineralized cartilage was seen in cortical bone of diaphysis in the fluoride-supplemented group. These results indicate that fluoride may affect the absorption of mineralized cartilage and bone as well as bone formation. Several possibilities have been inflicted for the effect of fluoride on the absorption of bone. It has been demonstrated that fluoride-treated bone is more resistant to resorption than untreated bone ${ }^{21)}$. The direct effect of fluoride to osteoclast is not well understood. In culture study, it showed a decrease in pit depth and inhibition of osteoclastic movement in the presence of fluoride ${ }^{22)}$. This is more likely to be consequence of a more stable fluoride- treated bone than any direct inhibitory effect of fluoride on osteoclasts.

In conclusion, 10 ppm fluoride supplement in drinking water caused changes in the calcified cartilage and mineralized bone in young rats.

\section{Acknowledgements}

This work was partially supported by grants in aid for scientific researches frpm the Ministry of Edition, Culture, Sports, Science and Technology (\#15209060,17406027, 17591910, 17591911, 1759179000).

\section{References}

1. Palmer C, Wolfe SH. Position of the American Dietetic Association: impact of fluoride on health. J Am Diet Assoc 105:1620-1628, 2005.

2. Rich C, Ensinck J. Effect of sodium fluoride on calcium metabolism of human beings. Nature 191:184-185, 1961.

3. Cheng PT, Bader SM, Grynpas MD. Biphasic sodium fluoride effects on bone and bone mineral: a review. Cell Mater 5:271 282, 1995.

4. Baylink DJ, Bernstein DS. The effects of fluoride therapy on metabolic bone disease. A histologic study. Clin Orthop Relat Res 55:51-85, 1967.

5. Meunier PJ. Treatment of vertebral osteoporosis with fluoride and calcium. Prog Clin Biol Res 332:221-230, 1990.

6. Riggs BL, Hodgson SF, O’Fallon WM, Chao EY, Wahner HW, Muhs JM, Cedel SL, Melton LJ 3rd. Effect of fluoride treatment on the fracture rate in postmenopausal women with osteoporosis. N Engl J Med 322:802-809, 1990.

7. Chachra D, Turner CH, Dunipace AJ, Grynpas MD. The effect of fluoride treatment on bone mineral in rabbits. Calcif Tissue Int 64:345-351, 1999.

8. Turner CH, Hasegawa K, Zhang W, Wilson M, Li Y, Dunipace AJ. Fluoride reduces bone strength in older rats. J Dent Res 74:1475-1481, 1995.

9. Farley JR, Wergedal JE, Baylink DJ. Fluoride directly stimulates proliferation and alkaline phosphatase activity of 


\section{J.Hard Tissue Biology.15(2): 61- 64,2006}

bone-forming cells. Science 222:330-332, 1983.

10. Grynpas MD, Hamilton E, Cheung R, Tsouderos Y, Deloffre P, Hott M, Marie PJ. Strontium increases vertebral bone volume in rats at a low dose that does not induce detectable mineralization defect. Bone 18:253-259, 1996.

11. Bonjour JP, Caverzasio J, Rizzoli R. Effect of fluoride on bone cells. Res Clin Forums 15:9-12, 1993.

12. Balena R, Kleerekoper M, Foldes JA, Shih MS, Rao DS, Schober HC, Parfitt AM. Effects of different regimens of sodium fluoride treatment for osteoporosis on the structure, remodeling and mineralization of bone. Osteoporosis Int 8:428-435, 1998.

13. Vaananen K, Zhao H. Osteoclast function: Biology and mechanisms. Principles of bone biology, ed. by Bilezikanen JP, Raisz LG, Rodan GA, Academic Press, San Diego, 2002, pp 127-140.

14. Grynpas MD. Fluoride effects on bone crystals. J Bone Miner Res 5:169-175, 1990.

15. Nyman JS, Reyes M, Wang X. Effect of ultrastructural changes on the toughness of bone. Micron 36:566-582, 2005.

16. Turner CH, Owan I, Brizendine EJ, Zhang W, Wilson ME, Dunipace AJ. High fluoride intakes cause osteomalacia and diminished bone strength in rats with renal deficiency. Bone 19:595-601, 1996.

17. Waddington RJ, Langley MS. Structural analysis of proteoglycans synthesized by mineralizing bone cells in vitro in the presence of fluoride. Matrix Biol 17:255-268, 1998.

18. Aoba T, Shimazu Y, Taya Y, Soeno Y, Sato K, Miake Y. Fluoride and apatite formation in vivo and in vitro. J Electron Microsc (Tokyo) 52:615-625, 2003.

19. Sogaard CH, Mosekilde L, Schwartz W, Leidig G, Minne HM, Ziegler R. Effects of fluoride on rat vertebral body biomechanical competence and bone mass. Bone 16:163-169, 1995.

20.Turner CH, Hinckley WR, Wilson ME, Zhang W, Dunipace AJ. Combined effects of diets with reduced calcium and phosphate and increased fluoride intake on vertebral bone strength and histology in rats. Calcif Tissue Int 69:51-57, 2001.

21. Grynpas MD and Cheng PT. Fluoride reduces the rate of disslotion of bone. Bone and minera 5:1-9, 1988.

22. Taylor ML, Boyde A, Jones SJ. The effect of fluoride on the patterns of adherence of osteoclasts cultured on and resorbing dentine: a 3-D assessment of vinculin-labelled cells using confocal optical microscopy. Anat Embryol (Berl) 180:427435, 1989. 